POS PROCEEDINGS

\title{
Construction of a Precision Four-Layer Floating Strip Micromegas Chamber
}

\author{
Jonathan Bortfeldt ${ }^{* a}$, Otmar Biebel ${ }^{a}$, Bernhard Flierl ${ }^{a}$, Felix Klitzner ${ }^{a}$, Philipp \\ Lösel $^{a}$, Ralph Müller ${ }^{a}$, André Zibell ${ }^{b}$ and Ralf Hertenberger ${ }^{a}$ \\ ${ }^{a}$ Ludwig-Maximilians-Universität Munich \\ ${ }^{b}$ Julius-Maximilians-Universität Würzburg \\ E-mail: jonathan.bortfeldtecern.ch
}

Floating strip Micromegas detectors have proven to be discharge sustaining and versatile high-rate capable tracking detectors. They exhibit a spatial resolution of $50 \mu \mathrm{m}$ and a single strip temporal resolution on the order of $5 \mathrm{~ns}$. Up to particle fluxes of $7 \mathrm{MHz} / \mathrm{cm}^{2}$ single particle tracking at an efficiency above $95 \%$ is possible. Recently we have further increased the high-rate capability by using alternative Neon based detector gases.

We constructed a four-layer floating strip Micromegas detector with an active area of $55 \times 33 \mathrm{~cm}^{2}$ subdivided into 768 strips per layer. Each readout plane consists of two separate printed circuit boards. The anode and cathode panels are realized as stiff aluminum honeycomb sandwich panels with $0.5 \mathrm{~mm}$ thick copper-clad, structured FR4 boards. The panels are assembled on a precisely planar table, that carries a high-precision alignment frame. The assembly procedure shall ensure the relative alignment of the two readout boards per layer and the correct inter-plane alignment. The construction and assembly procedures of the quadruplet chamber are presented. They can serve as a proof-of-concept study for the construction of large-area multi-layer Micromegas chambers. Planarity measurements with a laser distance sensor equipped coordinate measuring machine are discussed.

The European Physical Society Conference on High Energy Physics

22-29 July 2015

Vienna, Austria

${ }^{*}$ Speaker. 


\section{Introduction}

The presented works aims at demonstrating the feasibility of large-area floating strip Micromegas chambers. It is furthermore intended for investigating and qualifying the construction and assembly methods for large-area and multi-layer Micromegas based chambers. We are following the construction procedure foreseen for the Small Sector Module 2 of the ATLAS Muon New Small Wheel as our group is involved in the construction of this resistive strip Micromegas quadruplet chamber [1]. In order to reach the desired online spatial resolution of $100 \mu \mathrm{m}$ per layer, the overall module has to be built with precision better than $100 \mu \mathrm{m}$. The construction of the first full-size modules is ongoing.

Floating strip Micromegas were developed during the last years in order to minimize the influence of unavoidable discharges on the detection efficiency in Micromegas detectors. Completely avoiding resistive materials in the active area leads to an outstanding high-rate capability [2,3]. Recently low-material budget floating strip Micromegas with two-dimensional readout structure have been tested in carbon ion an proton beams at hit rates of up to $2 \mathrm{GHz}$.

In Fig. 1 a schematic of a floating strip Micromegas can be seen. Ionization charge, produced by traversing charged particles, is seperated in the $0.3 \mathrm{kV} / \mathrm{cm}$ electric drift field between cathode and micro-mesh. The electrons drift into the high-field amplification region between mesh and anode strip structure, where they are amplified in avalanche processes. Signals are collected on the anode strips and decoupled by congruent readout strips, individually connected to readout electronics. Charge densities exceeding $2 \times 10^{8} \mathrm{e} / \mathrm{mm}^{2}$ can lead to streamer forma-

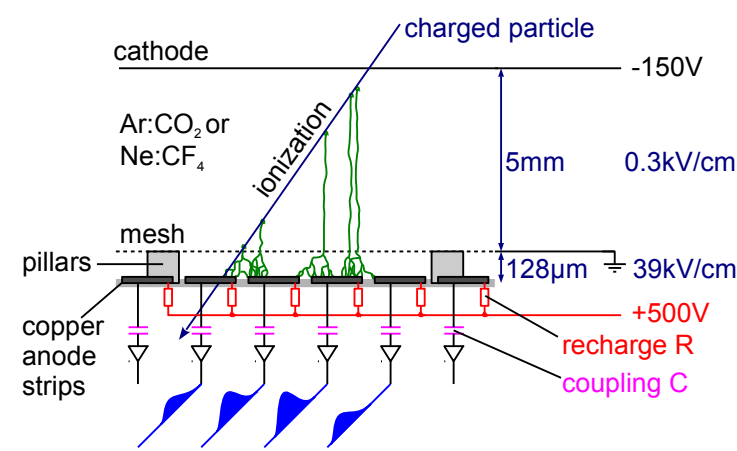

Figure 1: Schematic view of a floating strip Micromegas detector. tion, followed by discharges in the amplification region. During a discharge the potential of the affected floating strip adapts quickly to the mesh potential, quenching the discharge and leaving all other strips unaffected. Due to this major improvement, operating in highest-rate particle beams at excellent overall efficiency and constant gain is possible.

\section{Chamber Design \& Construction Method}

A cut through the floating strip Micromegas quadruplet chamber with overall outer dimensions of $414 \times 755 \times 75 \mathrm{~mm}^{3}$ is shown in Fig. 2. Readout and cathode structure are supported by stiff FR4-aluminum honeycomb sandwich panels, reinforced by aluminum bars. Additional aluminum and copper profiles are used for gas distribution and cooling, respectively. In order to facilitate cleaning and simplify commissioning, the stainless steel micro-mesh is not permanently attached to the mesh supporting pillars, but is glued onto a precision aluminum frame, that is screwed to the drift panel. 
The module consists of two double readout panels, carrying a readout structure on either side, one double drift panel, with cathode and micro-mesh on either side and two outer single drift panels. Each of the four readout layers features an active area of $534 \times$ $338 \mathrm{~mm}^{2}$, subdivided into 768 strips per layer with $440 \mu \mathrm{m}$ pitch. Each readout layer is formed by two individual printed circuit boards that have to be aligned precisely during assembly in order to guarantee an overall strip alignment better than $30 \mu \mathrm{m}$.

Planarity of the readout and drift panels is achieved by sucking the flex-

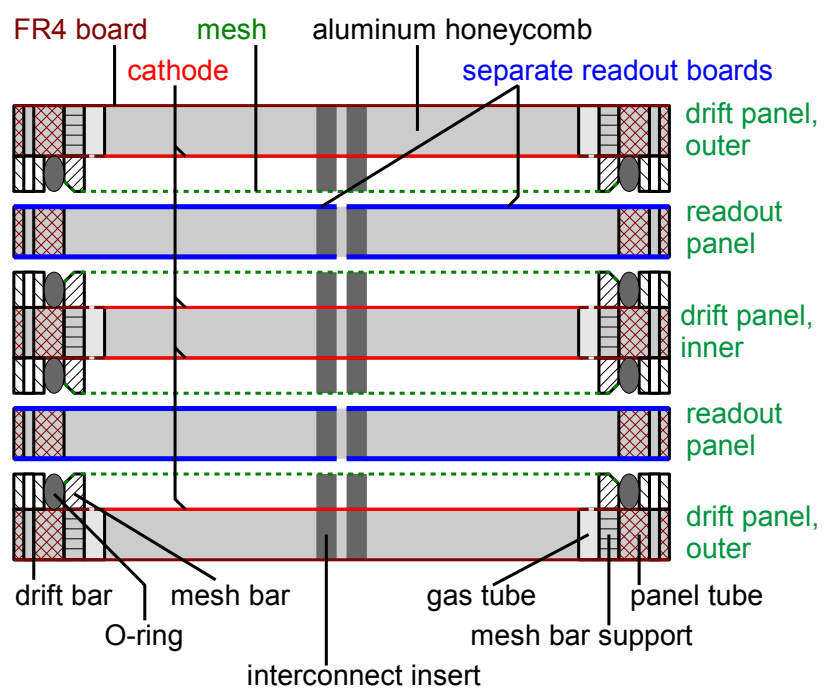

Figure 2: Cut through the Micromegas quadruplet. ible readout and cathode printed circuit boards (PCBs) during panel assembly to a light-weight table with an overall precision better than $30 \mu \mathrm{m}$ RMS. Aluminum bars and aluminum honeycomb with $9 \mathrm{~mm}$ cell-size are then glued on the PCBs. During glue curing, only little force is exerted onto the honeycomb and the bars in order to avoid internal stress build-up. This semi-panel is then transferred to a so called stiffback, a light-weight aluminum structure, with a surface planarity better than $10 \mu \mathrm{m}$ RMS. The semi-panel is sucked to the stiffback with 200 mbar under-pressure to ensure its planarity during the second gluing step. The second layer of PCBs is positioned and sucked to the table, a $300 \mu \mathrm{m}$ thick layer of glue is applied and the semi-panel, hanging on the stiffback, is placed on top.

After glue curing, the holes for module assembly, panel interconnection, gas distribution and mesh frame mounting are drilled in a single step on a CNC machine, without moving the panel. Before and after drilling, the planarity and the parallelicity of the panels is measured on a laser distance sensor equipped coordinate measurement machine with an overall accuracy of $10 \mu \mathrm{m}$.

The readout and drift panels are cleaned with solvent and high-pressure de-ionized water, dried and assembled vertically in a clean room.

\section{Readout Panel Assembly}

For the readout panels, the alignment of the readout PCBs within a single layer and also across the two sides of the panel should be better than $30 \mu \mathrm{m}$ to obtain the desired spatial resolution below $100 \mu \mathrm{m}$. The components of the readout panels are mechanically aligned with respect to two fixed precision cylinders during construction. This procedure will be described in the following. The planarity of both the readout and the drift panel should be better than $30 \mu \mathrm{m}$ RMS.

\subsection{Readout Board Preparation}

The readout structure is formed by a conventional multi-layer printed circuit board, on which the mesh-supporting pillar structure has been produced. The copper floating anode strips are guided 
out of the active region and are connected individually to positive high-voltage via screen-printed resistors. The congruent layer of readout strips is routed out of the active area on the opposite side and can be connected to readout electronics with soldered fine-pitch 130 pin connectors.
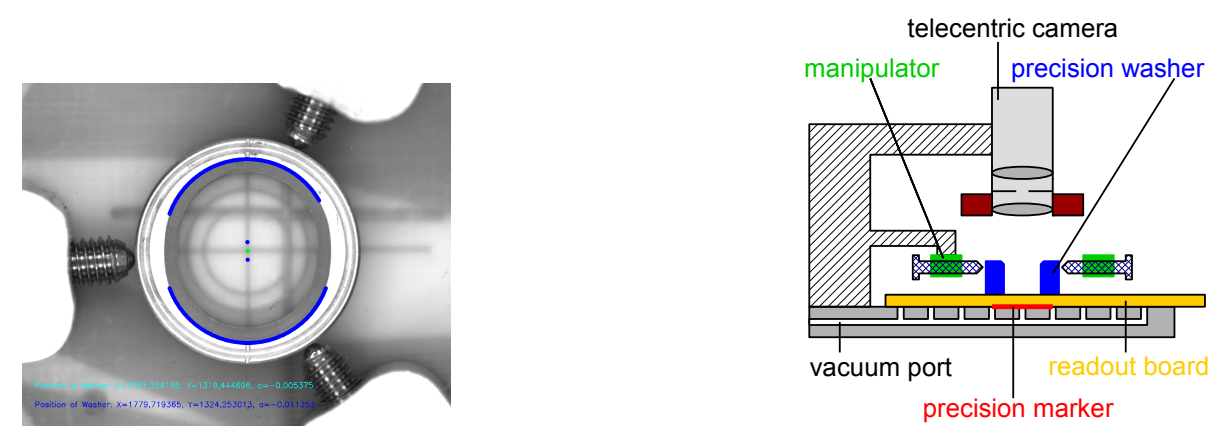

Figure 3: Circular and slot precision washers are glued onto the readout PCBs, aligned with respect to precision markers (left). Alignment is achieved based on position reconstruction from images acquired with a telecentric camera (right).

In the same production step as the floating strips, two precision markers (Fig. 3, left) are created on the readout PCBs, which thus have a precise position correlation with respect to the strips and can be used for optical alignment of the boards during construction. In order to enable a fully mechanical alignment during construction, as first step, slot and round brass washers with $8 \mathrm{~mm}$ diameter and $\mathrm{H} 7$ precision $^{1}$ are glued on the backside of the readout board, aligned with respect to the precision markers within $5 \mu \mathrm{m}$. Alignment between the washers and the precision markers is achieved by observing both with a telecentric camera and adjusting the washer position with a manipulator (Fig. 3, right).

\subsection{Assembly Procedure}

Two cleaned readout boards are placed on the table with the readout structure facing the table. Prealignment $\mathscr{O}(0.5 \mathrm{~mm})$ is achieved with aluminum distance pieces between a precision aluminum frame, permanently mounted to the table (Fig. 4, left), and the edges of the readout PCBs. A precision aluminum alignment frame, holding four $8 \mathrm{~mm} \mathrm{g6} \mathrm{dowel} \mathrm{pins}{ }^{2}$, is then placed on top. The dowel pins fit into the precision brass washers on the readout PCBs, thus precisely aligning the boards with respect to each other.

Absolute alignment on the table is achieved by pushing two precision surfaces on the alignment frame against two fixed $16 \mathrm{~mm}$ cylinders, held in place by the aluminum frame. The readout PCBs are then sucked to the table, the alignment frame can be lifted and the gap between the two readout PCBs is closed with Kapton tape. Two v-shaped and straight precision interfaces, similar to those on the alignment frame, are then glued to the lower PCB, accurately positioned with respect to the fixed precision cylinders.

A $300 \mu \mathrm{m}$ thick layer of Araldite 2011 is applied and structured with a notched trowel. Aluminum bars are placed into the glue and held in place by fixation fingers. Their position is defined by cylindric distance pieces between the bars and the surrounding aluminum frame (Fig. 4, right).

\footnotetext{
${ }^{1}$ corresponding to $\left(8.000_{-0.000}^{+0.015}\right) \mathrm{mm}$

${ }^{2}$ corresponding to $\left(8.000_{-0.014}^{-0.005}\right) \mathrm{mm}$
} 

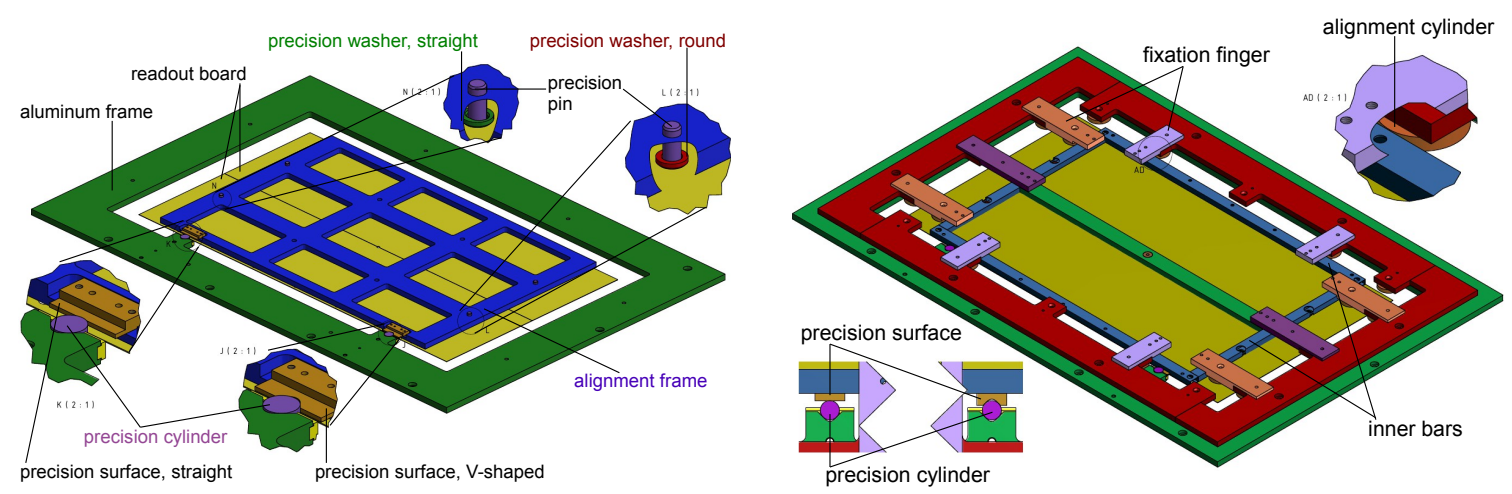

Figure 4: Left: Absolute alignment of the two readout PCBs (yellow) is achieved with the removable alignment frame (blue), the aluminum frame (green) is mounted on the table. Right: After alignment, two v-shaped and straight precision interfaces (orange) are glued onto the lower PCB. After applying glue, aluminum bars and aluminum honeycomb (not shown) are placed onto the PCBs and held in place by alignment cylinders and fixation fingers.

Aluminum honeycomb pieces are placed between the bars and a peek interconnection insert is placed in the center of the panel. A $20 \mathrm{~mm}$ wooden plate, loaded with about $30 \mathrm{~kg}$ lead, is put on top to guarantee a good contact between the honeycomb and the PCBs during glue curing. In a second gluing step two copper cooling channels are glued on the two short sides of the readout panel. After curing, this semi-panel is transferred to a light-weight stiffback and sucked against it, ensuring the planarity of the still flexible semi-panel.

The described alignment procedure is repeated with the readout PCBs for the second layer, glue is applied and the semi-panel, sucked to the stiffback is placed on top. The semi-panel is aligned with respect to the precision cylinders using the two precision surfaces, that have been glued to the first gluing side. This procedure ensures an accurate relative alignment of the two readout panel sides. The parallelicity of both readout layers during glue curing is defined by four precision steel distance pieces between table and stiffback.

\subsection{Milling \& Drilling Procedure}

Holes needed for assembly and panel interconnection are drilled into the readout panel after gluing in a three step process. Additionally four $10 \mathrm{~mm} \mathrm{H7}$ holes are milled in the four corners of the readout panel representing a possibility for relative alignment of readout and drift panels during assembly.

The panel is positioned on a CNC milling machine using the precision interfaces in the panel. A $0.8 \mathrm{~mm}$ deep counterbore is produced for all holes with a by $1 \mathrm{~mm}$ larger diameter than the final hole. The panel is flipped over and the procedure is repeated. Finally all through-holes are produced in a single step, without moving the panel. This ensures the relative alignment of all holes with machine precision.

\section{Drift Panel Assembly}

The alignment of the two sides of drift panels is not as essential as for the readout panels, an 
overall alignment of bars and PCBs $\mathscr{O}(0.5 \mathrm{~mm})$ is sufficient. The alignment during construction thus relies on distance pieces between the aluminum frame on the table and the bars and PCBs.

\subsection{Assembly Procedure}

The assembly procedure is similar to that of readout panels, cf. section 3.2. The cathode PCB is placed on the table, alignment is done via three distance pieces between the aluminum frame on the table and the PCB edges. The board is sucked to the table, for vacuum distribution a stainless steel micro-mesh with $90 \mu \mathrm{m}$ wires is used.

Aluminum bars for mounting, as support of the mesh frames and for gas distribution are placed onto the PCB into a $300 \mu \mathrm{m}$ thick layer of Araldite 2011, cf. Fig. 2. They are held in place by fixation fingers and cylindric distance pieces with respect to the outer aluminum frame. Aluminum honeycomb is placed between the bars, the peek interconnection insert is positioned in the central aluminum bar, a plate with about $30 \mathrm{~kg}$ weight presses the honeycomb into the glue during curing. After curing, the semi-panel is transferred to a stiffback, the PCB for the second gluing side is placed, glue is applied, and the semi-panel on the stiffback is lowered into the glue. The alignment between the two layers is achieved by three cylindric distance pieces between the aluminum bars in the semi-panel and the aluminum frame on the table. The parallelicity between the two layers is ensured, as before, by precision steel distance pieces between the table and the stiffback.

\subsection{Milling \& Drilling Procedure}

The drilling and milling is done in the same way as for the readout panel, cf. section 3.3. Additionally $1.5 \mathrm{~mm}$ holes for gas distribution and M3 thread holes for screwing of the mesh bars are produced. The alignment of the panel is done with respect to the sides of the aluminum bars in the panel. As before, all holes through the aluminum are produced in a single step, without moving the panel, ensuring the precise relative alignment of all holes.

\subsection{Achieved Planarity}

The planarity of the central double drift panel has been measured before and after drilling with a laser distance sensor on a coordinate measurement machine. No significant differences of the planarity in the active region before and after drilling are observed. The topology of the panel after drilling, simply laying on the table during the measurements, is shown in Fig. 5. The panel shows a slight overall bent towards the first gluing side with a maximum deviation from the plane by approximately $20 \mu \mathrm{m}$. The planarity of the both sides has a variation of $9.6 \mu \mathrm{m}$ and $11.3 \mu \mathrm{m}$ RMS respectively, which is well within the desired range.

The panel is in the lower left corner approximately $30 \mu \mathrm{m}$ thicker than in the upper right corner (Fig. 5, right), the parallelicity has a variation of $11.7 \mu \mathrm{m}$ RMS, which is again well within the desired region.

\section{Method for Quadruplet Assembly}

\subsection{Mesh Gluing}

The mesh bars (cf. Fig. 2) are screwed onto the drift panel. A peek interconnection distance piece is glued onto the cathode, aligned with the interconnection hole. The micro-mesh is stretched 

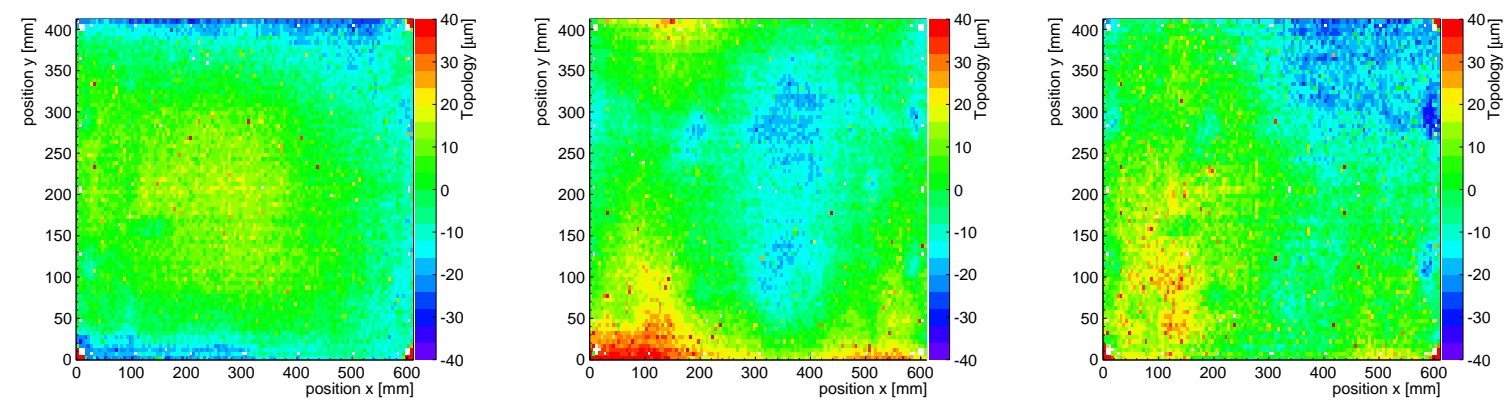

Figure 5: Measured planarity of the first (left) and the second gluing side (center) of the double drift panel after drilling and threading. The measured parallelicity of both sides is shown on the right.

using pneumatic clamps to a tension of $(11 \pm 1) \mathrm{N} / \mathrm{cm}$ and glued to an aluminum transfer frame. It is cleaned with NGL solvent, scrubbed and rinsed with high-pressure, de-mineralized water. After drying it is placed on top of the drift panel and glued to the inclined side of the mesh bars. The excess mesh is cut with a scalpel.

\subsection{Assembly Procedure}

The panels are cleaned in the same way as the mesh, dried and assembled vertically into a quadruplet in the clean room. The alignment of the two readout panels can be achieved by placing the respective precision surfaces in each panel onto two precision rails, screwed to an aluminum plate that is defining their distance accurately. The alignment of the drift panels is done via the mounting holes in all panels, this yields a sufficient precision. As an alternative alignment method, the $10 \mathrm{~mm} \mathrm{H7}$ holes in all panels can be used. The mutual distance of the panels and with it the drift gap width is defined by precision-ground aluminum bars with a thickness of $5.15 \mathrm{~mm}$, with maximum variations below $\pm 0.1 \mathrm{~mm}$. Gas tightness is ensured by a soft $6 \mathrm{~mm}$ O-ring. The panels are screwed together at the sides and in the center.

\section{Summary}

We are constructing a floating strip Micromegas quadruplet with an active area of $55 \times 33 \mathrm{~cm}^{2}$ per layer and an overall precision better than $100 \mu \mathrm{m}$. The construction, milling and assembly methods have been developed and tested. Several drift panels have been assembled with an accuracy well within the desired range. The assembly of the two readout panels is ongoing.

\section{References}

[1] ATLAS Collaboration, New small wheel technical design report, Tech. Des. Rep. CERN-LHCC-2013-006, CERN, Geneva, 2013.

[2] J. Bortfeldt, The Floating Strip Micromegas Detector. Springer Theses. Springer, 2015.

[3] J. Bortfeldt, M. Bender, O. Biebel, H. Danger, B. Flierl, R. Hertenberger, P. Lösel, S. Moll, K. Parodi, I. Rinaldi, A. Ruschke and A. Zibell, High-rate capable floating strip micromegas, Nuclear Physics B Proceedings Supplement (2015). 